\title{
The role of microRNA in the resistance to treatment of hepatocellular carcinoma
}

\author{
Muhammad Yogi Pratama ${ }^{1,2}$, Devis Pascut ${ }^{1}$, Muhammad Nasrum Massi $^{2}$, Claudio Tiribelli ${ }^{1}$ \\ ${ }^{1}$ Fondazione Italiana Fegato, AREA Science Park Bazovizza, Trieste, Italy; ${ }^{2}$ Faculty of Medicine, Universitas Hasanuddin, Makassar, Indonesia \\ Contributions: (I) Conception and design: MY Pratama, D Pascut, C Tiribelli; (II) Administrative support: C Tiribelli; (III) Provision of study \\ materials or patients: None; (IV) Collection and assembly of data: MY Pratama; (V) Data analysis and interpretation: None; (VI) Manuscript writing: \\ All authors; (VII) Final approval of manuscript: All authors. \\ Correspondence to: Muhammad Yogi Pratama. Associate Researcher, Fondazione Italiana Fegato, AREA Science Park Bazovizza, 34149 Trieste, Italy. \\ Email: yogi.pratama@fegato.it.
}

\begin{abstract}
Hepatocellular carcinoma (HCC) is the second most common cause of cancer-related death with a limited efficacy of treatment for intermediate and advanced stages of the disease. Several therapeutic approaches such as trans-arterial chemoembolization (TACE) with anthracyclines, cisplatin and multikinase inhibitor sorafenib have been appealing choices of treatments yet failed to reach a satisfactory outcome mainly due to the numerous mechanisms that influence patient's response. MicroRNAs (miRNAs) are key regulators of many intracellular processes related to drug resistance. This phenomenon has been linked to the modulation of several complex pathways, ranging from the loss of ability of drug accumulation, protective mechanism of autophagy, adaptive mechanism of cancer cells towards the drugs-induced environment, decrease DNA damage and suppression of downstream events that transduce its signal into apoptosis. We summarize the recent findings on the involvement of miRNAs in various drug resistance-related mechanisms in the development of resistance to anthracyclines, cisplatin and sorafenib therapies. Furthermore, we describe the possible application of miRNAs as circulating biomarkers predicting therapy response in HCC. Thus, the undeniable potential and paramount role of miRNA in drug resistance may eventually lead to improved clinical strategies and outcomes for HCC patients.
\end{abstract}

Keywords: Anthracyclines; drug resistance; hepatocellular carcinoma (HCC); microRNA (miRNA); sorafenib

Submitted Sep 03, 2019. Accepted for publication Sep 23, 2019.

doi: $10.21037 /$ atm.2019.09.142

View this article at: http://dx.doi.org/10.21037/atm.2019.09.142

\section{Introduction}

Over the years, there are significant developments in the therapeutic approaches of cancer after the introduction of several drugs able to improve the survival and overall prognosis of cancer patients. However, there are limitations regarding the efficacy of these drugs, mainly associated with the complicated nature of cancer cells and the multifactorial mechanism of drug resistance.

Hepatocellular carcinoma (HCC) is one of the most common malignancies worldwide (1). It is primarily known as the second most common cause of cancer-derived mortality, contributing to 746,000 deaths in 2012 (9.1\% of the total) (1). The late occurrence, principally in advanced liver disease, make HCC as a "difficult to treat" cancer, as surgical approach and liver transplantation, the only radical treatment for the disease, are feasible only in the early stages (2).

For intermediate and advanced stages, several therapeutic approaches are available according to the degree and severity of cancer. Trans-arterial chemoembolization (TACE) is the most widely used primary treatment for unresectable HCC, as a palliative or downstaging purposes. TACE combines the cytotoxic effect of several drugs (cisplatin or doxorubicin) and ischemic effect from the embolization of the branches of a hepatic artery, inducing necrosis of the tumoral tissue $(2,3)$. Even though 
previous studies have established the survival advantage of TACE, recent data have challenged this conclusion (4). A complete response failed to reach significant value either in bland embolization method or drug-eluting bead (DEB)-TACE (5), in addition recurrence episodes are still significantly high (6-8).

Molecular-targeted therapies for HCC has been an appealing option since the development of the multikinase inhibitor sorafenib, firstly described with the SHARP trial in 2007 in a cohort of advanced HCC $(2,9,10)$. Sorafenib is an inhibitor of Raf-kinase and several tyrosine kinases receptors implicated in tumorigenesis, tumor progression, and vascularization. In most tumor types, sorafenib induces apoptosis by down-regulating the anti-apoptotic protein Mcl-1 through a MEK/ERK-independent mechanism $(11,12)$. Sorafenib is also described to target pro-angiogenic and pro-fibrotic players such as receptor tyrosine kinase (c-Kit), Fms-like tyrosine kinase (FLT-3), vascular endothelial growth factor receptor (VEGFR), platelet-derived growth factor receptor (PDGFR- $\beta$ ), and other tyrosine kinases $(13,14)$. Unfortunately, even though sorafenib was an achievement for HCC therapy, the outcomes are still far from being satisfactory (15).

The understanding towards refractory to either chemotherapeutic drugs or targeted therapy is needed to identify the key players involved in the resistance. Thus, it is essential to understand cellular pathways involved in the mechanism of resistance.

\section{MicroRNA (miRNA) in cancer}

Since their discovery in C. elegans in 1993, miRNAs have drawn a great deal of interest among researchers, especially in consideration of their role in post-transcriptional gene regulation (16). They are a class of highly conserved short non-coding RNAs, 18 to 25 nucleotides long, which regulate the expression of various genes. It is hypothesized that miRNAs can regulate more than $60 \%$ of the proteincoding genes in cells $(16,17)$ with an existing overlap among miRNAs targeting the same mRNA, as well as among different mRNAs targeted by one single miRNA (18).

Only in 2002, miRNAs entered the medical research with the report of their involvement in pathological conditions, including cancer (19). Abundant miRNA profiling studies described the significant difference of miRNA expression in tumor samples when compared to healthy tissues. The relationship between decreased miRNA expression and the consequently increased expression of the oncogenic target genes became evident with the identification of RAS as a cellular target of let-7 in human cancer, suggesting a tumorsuppressor function for some miRNAs (20). Conversely, the increase of specific miRNAs in tumor cells indicate their role as oncogenes $(21,22)$, as for the cluster miR-17-92 found to be frequently amplified in B-cell lymphomas (22). Following the first miRNA profile investigation in HCC in 2006 (23), more than 2,000 studies have focused on the role of miRNAs in this disease. Currently, several, although incomplete information exists about the abnormal regulation of miRNAs in HCC and several studies reported the involvement of miRNAs in multi-drug resistance linking them to poor prognosis.

\section{miRNAs in drug resistance in HCC}

In tumor resistant cells, several mechanisms participate in the development of drug resistance in HCC. Recently, it was shown that altered expression of subsets of miRNAs plays an essential role in regulating genes involved in cell apoptosis, cell proliferation, autophagy, epithelial-mesenchymal transition (EMT) and regulation of drug efflux.

Although these mechanisms have been previously investigated, the role of miRNAs remains ambiguous and unclear. However, there were several in vitro studies reporting the involvement of specific miRNAs in each different pathway related with drug resistance in different HCC cell lines, suggesting their important role as a critical player in both anthracyclines and sorafenib resistance (Tables 1,2). In this review, we will clarify the role of these regulatory RNAs in the onset of drug resistance in HCC, focusing on the widely used therapeutic drugs against the liver tumor.

\section{miRNAs and their involvement in the mechanisms of drug resistance}

It is hypothesized that doxorubicin (dox) resistance results from the loss of ability for the drug to accumulate in the nucleus, decrease DNA damage, and suppression of the downstream events that transduce the DNA damage signal into apoptosis (47). The mechanism of resistance to sorafenib involves several complex genetic and epigenetic alterations, and it is assumed that miRNAs play a role in regulating resistance via the involvement in some canonical drug-resistance pathways (14). Primary resistance is assumed to be derived from the genetic heterogeneity of HCC, even though the exact mechanism remains unclear (14). 
Table 1 miRNAs involved in anthracyclines and cisplatin resistance-related pathways

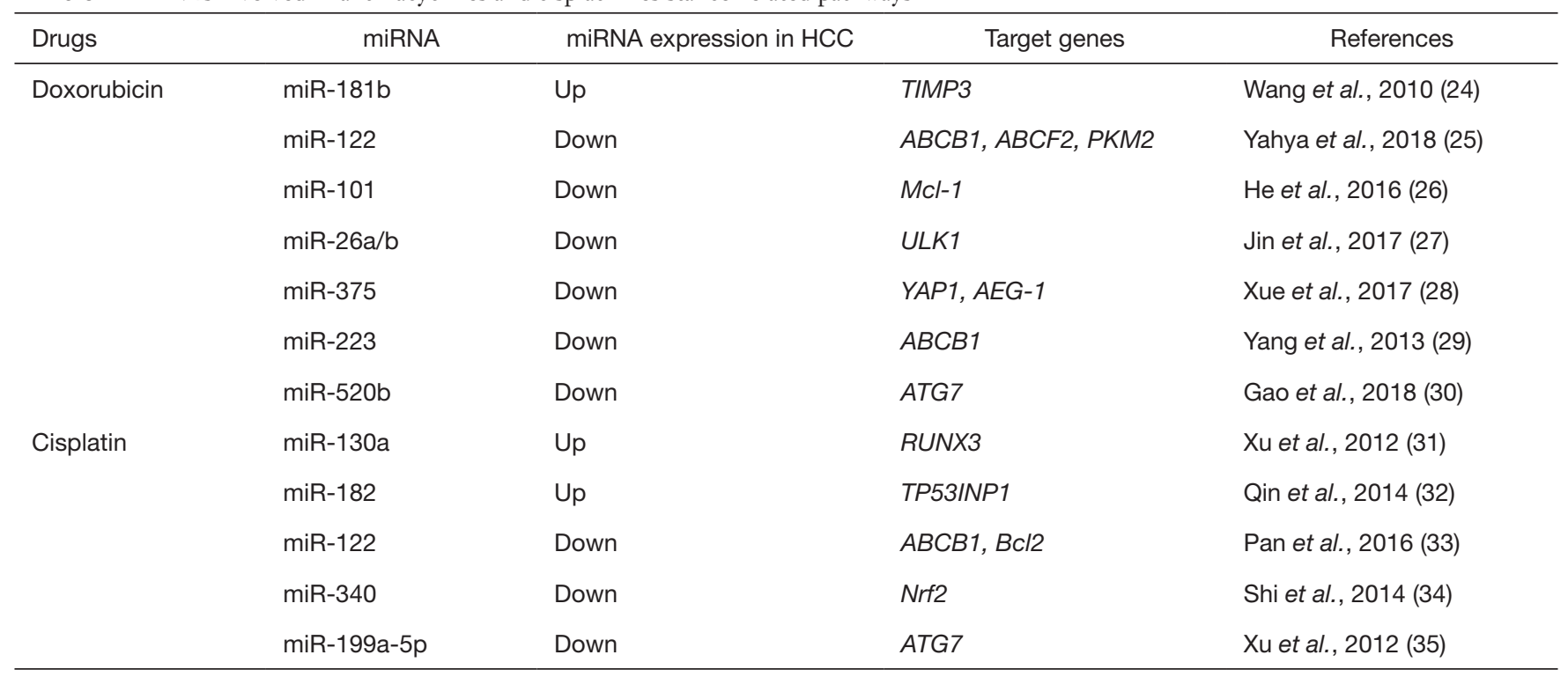

Table 2 miRNAs involved in sorafenib-induced resistance

\begin{tabular}{|c|c|c|c|c|}
\hline Drugs & miRNA & miRNA expression in $\mathrm{HCC}$ & Target genes & References \\
\hline \multirow{12}{*}{ Sorafenib } & miR-222 & Up & PTEN & Liu et al., 2015 (37) \\
\hline & miR-21 & Up & $A K T$ & Tang et al., 2016 (38) \\
\hline & miR-153 & Up & & \\
\hline & miR-217 & Up & & \\
\hline & miR-494 & Up & & \\
\hline & miR-10a-5p & Up & & \\
\hline & miR-494 & Up & PTEN, P27, PUMA & Pollutri et al., 2018 (39) \\
\hline & miR-122 & Down & $I G F-1 R$ & Xu et al., 2015 (42) \\
\hline & miR-137 & Down & ANT2 & Lu et al., 2017 (43) \\
\hline & miR-34a & Down & $B c / 2$ & Yang et al., 2014 (44) \\
\hline & MiR-338-3p & Down & $H I F-\alpha$ & Xu et al., 2014 (45) \\
\hline & miR-193b & Down & $M c /-1$ & Mao et al., 2014 (46) \\
\hline
\end{tabular}

\section{Multidrug resistance transporters}

One of the most important mechanisms of dox resistance consists of drug elimination by the transporter family known as the ATP-binding cassette (ABC) transporters, known to be highly abundant in hepatocytes (47). ABCB1, also known as multidrug resistance 1 (MDR1) or P-glycoprotein (P-gyp), is expressed in $80-90 \%$ of HCC cases $(25,48,49)$ 


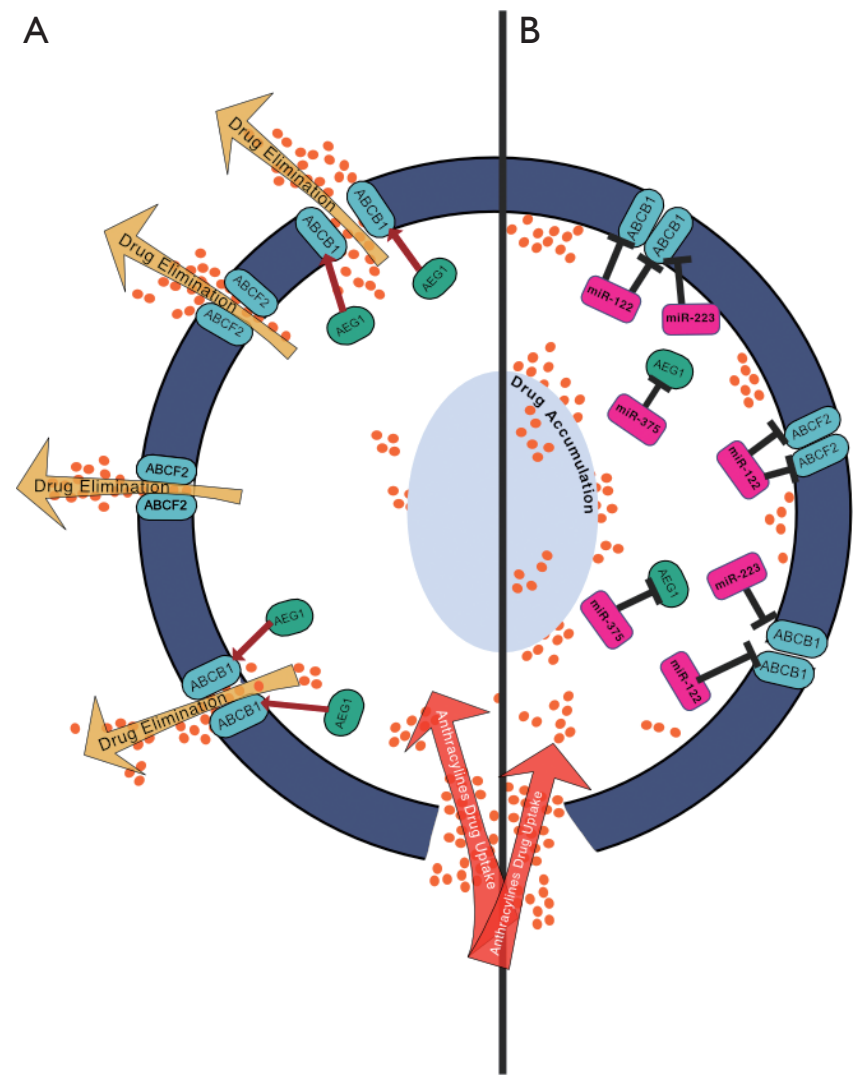

Figure 1 Mechanisms of drug accumulation in the cell. (A) miRNAs intracellular drug concentration by working as pump actively extruding drug compounds leading to resistance of HCC cells; (B) miRNAs involved in inhibiting MDR genes, leading to the accumulation of anthracyclines inside of HCC cells. HCC, hepatocellular carcinoma; MDR, multi-drug resistance transporter.

where it lowers the intracellular drug accumulation by working as a pump actively extruding exogenous compounds out of cells $(25,48,50)$. Other ABC family members, such as $\mathrm{ABCB} 1, \mathrm{ABCC} 1, \mathrm{ABCC} 2$, and $\mathrm{ABCG} 2$ are also expressed in HCC, and the overlap in the substrate recognition makes those transmembrane transporters as one of the primary causes of drug failure (Figure 1).

Although several miRNAs are known to target different $\mathrm{ABC}$ transporters in HCC (51), only few of them were experimentally linked to anthracyclines resistance in this tumor (Figures 2,3). miR-122, one of the most abundant miRNA expressed in the liver, is known to be downregulated in HCC (52). This miRNA plays an essential role in hepatic lipid metabolism, hepatocyte development, and differentiation $(53,54)$. miR-122 suppresses the anti-apoptotic gene $\mathrm{Bcl}-2$ and regulates the expression of pyruvate kinase M2 (PKM2), involved in the proliferation and ability for the tumor cells to preserve
ATP, that is needed for chemo-resistant cells to survive under stress $(33,55)$. The overexpression of miR-122 induces cell cycle arrest that leads to the down-regulation of multidrug-resistant (MDR) genes, ABCB1, and ABCF2 $(25,55)$. ABCB1 gene is also directly targeted by miR-223 in both mRNA and protein levels, and the overexpression of miR-223 can sensitize HCC cells to dox (29). Moreover, miR-375 is also able to decrease the expression of ABCB1 by targeting astrocyte elevated gene-1 (AEG-1) and thus participating in dox resistance $(28,56,57)$. Recent evidence suggests the involvement of miR-590-5p on dox resistance. miR-590-5p have been proven to target yes-associated protein 1 (YAP1) that promotes cancer resistance by upregulating $\mathrm{ABC}$ transporters (58). The overexpression of this miRNA chemosensitizes resistant cells both in vitro and in vivo interfering with the YAP1 network (58). This miR-590-5p/YAP1 axis was correlated with chemotherapy response in HCC patients. Although the few clinical 


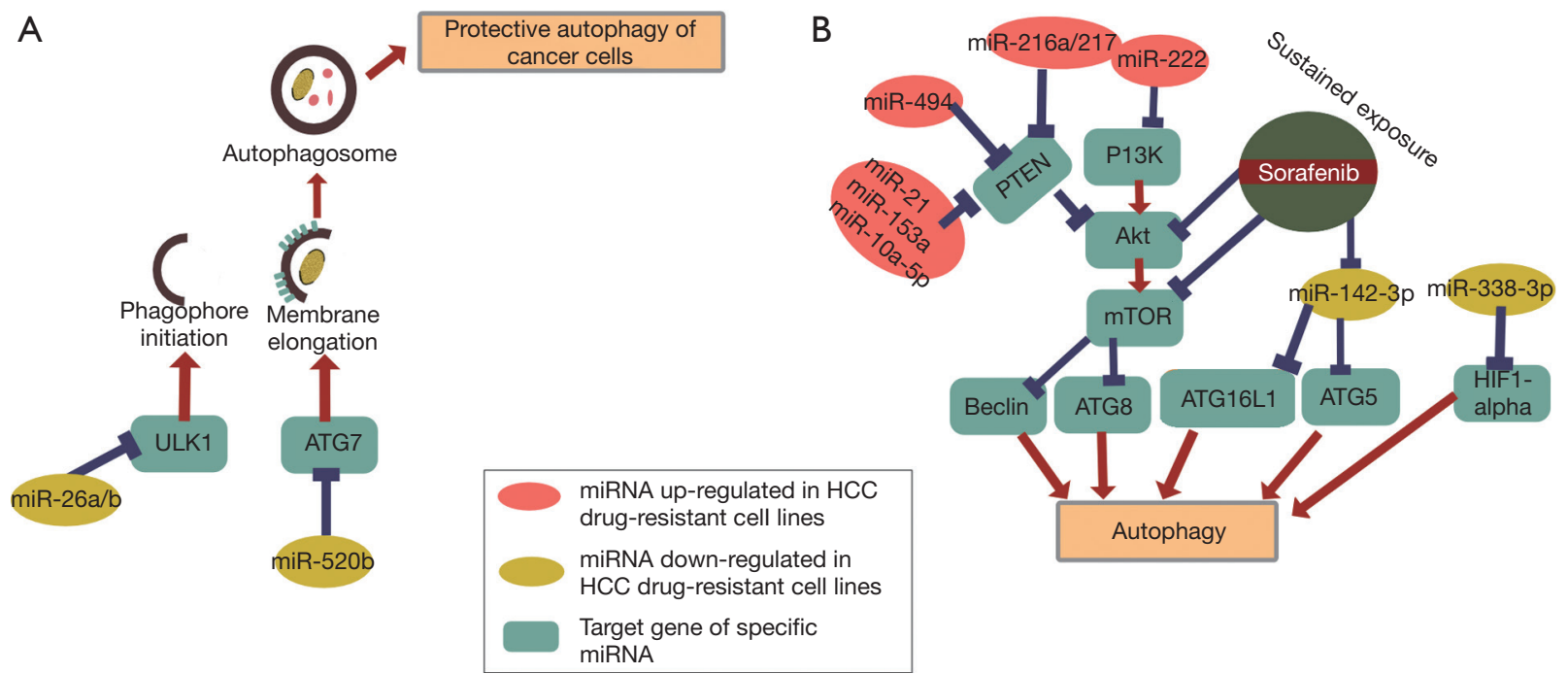

Figure 2 miRNAs involved in the multiple pathways of autophagy in cancer cells that develop resistance to anthracyclines and cisplatin (A) and sorafenib (B).

specimens analyzed, the YAP1 was higher in drug-resistant patients while miR-590-5p was downregulated, providing new insights into the identification of molecular targets for co-adjuvant therapies. Similar with miR-509-5p, the involvement of miR-122, miR-223, and miR-375 in the MDR1 drug-resistance related pathway $(29,33,56)$ show a remarkable potential to re-sensitize cells to dox- resistance, becoming a new strategy combining miRNA therapeutics with chemotherapy.

\section{Autophagy}

Autophagy is an adaptive mechanism promoting cell homeostasis by the degradation of useless and damaged proteins or other cytoplasmic components in the lysosomal system $(59,60)$. It is assumed to provide a protective effect against cancer, as it functions to prevent neoplastic transformation by removing damaged organelles and proteins complexes from cells exposed to stressful and pro-oncogenic conditions (61). However, in tumor cells, autophagy will also maintain metabolic homeostasis when cancer cells are subjected to stressful environments such as nutrient deprivation, hypoxia, or drug-induced damages during chemotherapy or targeted therapy (62). Several pathways, such as the mammalian target of rapamycin (mTOR) pathway, EFGR/Ras/MAPK pathway, p53 pathway, and hypoxia-inducible factor-1 (HIF-1) signaling pathway are primary regulators of autophagy in cancer cells (63). Furthermore, the hypoxic-environment after an anti-angiogenic therapy promotes HIF-1-mediated autophagy (63). However, depending on the type of anticancer treatment and genetic context of cancer, autophagy might also induce different effect and conversely work as an inducer of autophagic cell death, a mechanism that remains poorly explored and controversial (62).

Recently, the Unc-51 like autophagy activating kinase (ULK1), a serine/threonine protein kinase responsible for autophagy initiation (64) was identified as a target of the miR-26 family (miR-26a and miR-26b) (27). In doxtreated HepG2 cells, ULK1 was increased highlighting the protective role of autophagy in cancer cells. Interestingly, the overexpression of miR-26a/b decreased the expression of the ULK1 protein and sensitizing cancer cells to dox both in vitro and in vivo (27).

Several miRNAs involved in two protein conjugation systems that are required for autophagosome elongation and maturation in autophagy (65). One of the systems involved autophagy-related gene 7 (ATG7) that is targeted by miR-375, preventing the maturation of authophagosome thus inhibiting autophagy (66). Even though miR-375 was not directly involved in drug resistance, it is the inhibition of its target ATG7 that linked this miRNA to drug resistance (30). Another important miRNA in this pathway is miR-520b that is known to regulate ATG7 in both clinical and experimental settings. The down-regulation of miR-520-b and the up-regulation of its target ATG7 was 
A
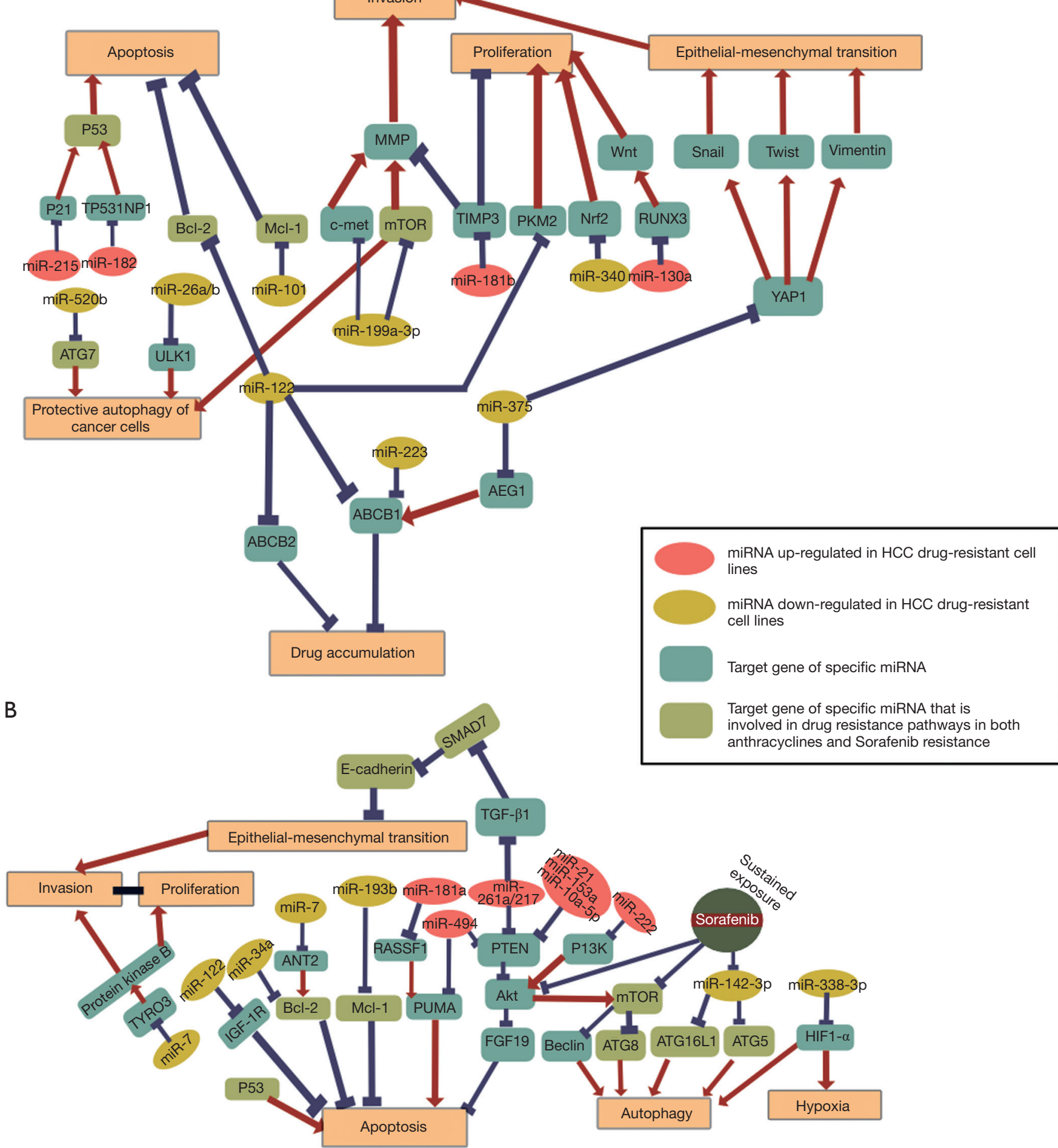

Figure 3 Multiple interpolated pathways regulated by miRNAs in the resistance to anthracyclines (A) and sorafenib (B) in HCC. Furthermore, several miRNAs target numerous genes while several genes are known to be involved either in both anthracyclines and sorafenib resistance. HCC, hepatocellular carcinoma. 
observed both in human HCC tissues and dox-resistant HCC cell lines (30); indeed the resistance was reversed after restoring the expression of miR-520b using ectopic miR$520 \mathrm{~b}$ mimic (30).

The role of autophagy during treatment with sorafenib is paradoxical. Some studies have established that sorafenibinduced autophagy is a cellular adaptive mechanism that promotes the survival of cancer cells (67). Thus inhibition of autophagy will enhance the effect of the drug (68). On the contrary, sorafenib was reported to inhibit the prodeath role of dox-induced autophagy in Hep3B cells (69-71). In sorafenib-resistant HCC cells, miR-142-3p was able to sensitize cells to the drug by inhibiting the druginduced autophagy (72). It was demonstrated that sorafenib significantly reduced miR-142-3p levels by acting on the transcription factor PU.1. However, the overexpression of miR-142-3p was able to prevent the cytoprotective autophagy that protects cancer cells from sorafenib by targeting two key autophagy-related proteins, ATG5, and ATG16L1 (72). This mechanism might be one prospective therapeutic strategy to prevent cytoprotective autophagy and overcome sorafenib resistance (72).

Conversely, other evidence showed the opposite role of autophagy in inducing cell death. The inhibition of crucial autophagy regulators such as PTEN and AKT/ mTOR pathway in HCC cells was described on decreasing the sensitivity of cells to sorafenib (73). PTEN is a tumorsuppressing phosphatase that inhibits Akt activation and thus preventing mTOR upregulation and the consequent regulation of the autophagic proteins LC3 and Beclin-1 (74). One study described that miR-21 is highly expressed in sorafenib-resistant cell lines and inhibiting autophagy by targeting PTEN (73). Furthermore, in vivo experiment by injecting anti-miR-21 oligonucleotides along with Sorafenib reduced the size of the tumor, pointing to the potential of anti-miR-2 1 as a prospective adjuvant therapy to overcome sorafenib resistance (73). Despite the findings in cell models, serum miR-21 was not able to predict sorafenib response in HCC patients (75).

Additional studies described the correlation between miRNA and autophagy-related sorafenib resistance; miRNAs such as miR-153, miR-216a, miR-217, miR-10a$5 \mathrm{p}$, miR-222, negatively regulate PTEN, leading to the overexpression of AKT and mTOR, regulating autophagy, proliferation, and apoptosis (36-38). miR-494 also regulates the AKT/mTOR pathway and increases cell survival during stress condition induced by sorafenib, suggesting its involvement in the mechanism of autophagy activated during stress condition $(39,65)$.

Collectively, the role of autophagy in promoting or preventing Sorafenib resistance is not yet fully clarified and needs more investigation. Disclosing the miRNA networks that participate in the regulation of autophagy (Figures 2,4) gives further hints to alternative therapeutically strategies.

\section{Hallmarks of cancer}

\section{EMT}

EMT is known to be an essential mechanism in the initial steps of cancer invasion and metastasis, as well as in the acquired resistance to cell senescence and apoptosis (76). It is a process where epithelial cells lose the polarity and cellcell adhesion turning into an invasive mesenchymal cells' phenotype. Cells undergoing EMT are characterized by the activation of zinc-finger transcriptional repressors that are responsible of the decreased expression of E-cadherin, an epithelial gene that functions as a full EMT inducer and crucial step in the progression of cancer invasion $(77,78)$. The down-regulation of transforming growth factor- $\beta$ (TGF- $\beta$ ) found in up to $40 \%$ of HCC, is recognized as a crucial event that promotes EMT and enhances the migratory and invasive properties of cancer cells by involving the transcriptional regulators such as SMAD family $(79,80)$. Moreover, EMT results in the dysregulation that involves TGF- $\beta 1$ and Yes-Associated Protein 1 (YAP1) (81). Recently, the identification of miR-375 as a YAP1 antagonist in cell models has suggested a complex network connecting EMT, apoptosis and $\mathrm{ABC}$ transporters modulation in drug resistance (Figure 3, Figure $5 A$ ) $(28,56,57)$.

The long-term exposure to sorafenib leads to EMT in sorafenib-resistant HepG2 and HuH7 cells resulting in the loss of E-cadherin and a concomitant increase in the cell growth and metastatic potential (82). E-cadherin is also suppressed by the transcription factor SNAIL induced by the serum response factor (SRF), a member of the MADSbox family resulting in a reduction of cytotoxic effects of sorafenib in cancer cells (83). Furthermore, both SRF and SNAIL were overexpressed in advanced HCC (83).

The miR-216a/217 cluster was significantly upregulated in HCC tissues from patients with recurrent disease (36). Further experiments in HCC cell lines demonstrated the association of this miRNA cluster with EMT phenotype by targeting SMAD7, an antagonist of TGF-B type 1 receptor (36). The over-expression of these miRNAs in cellular models leads to TGF- $\beta$ pathway activation 

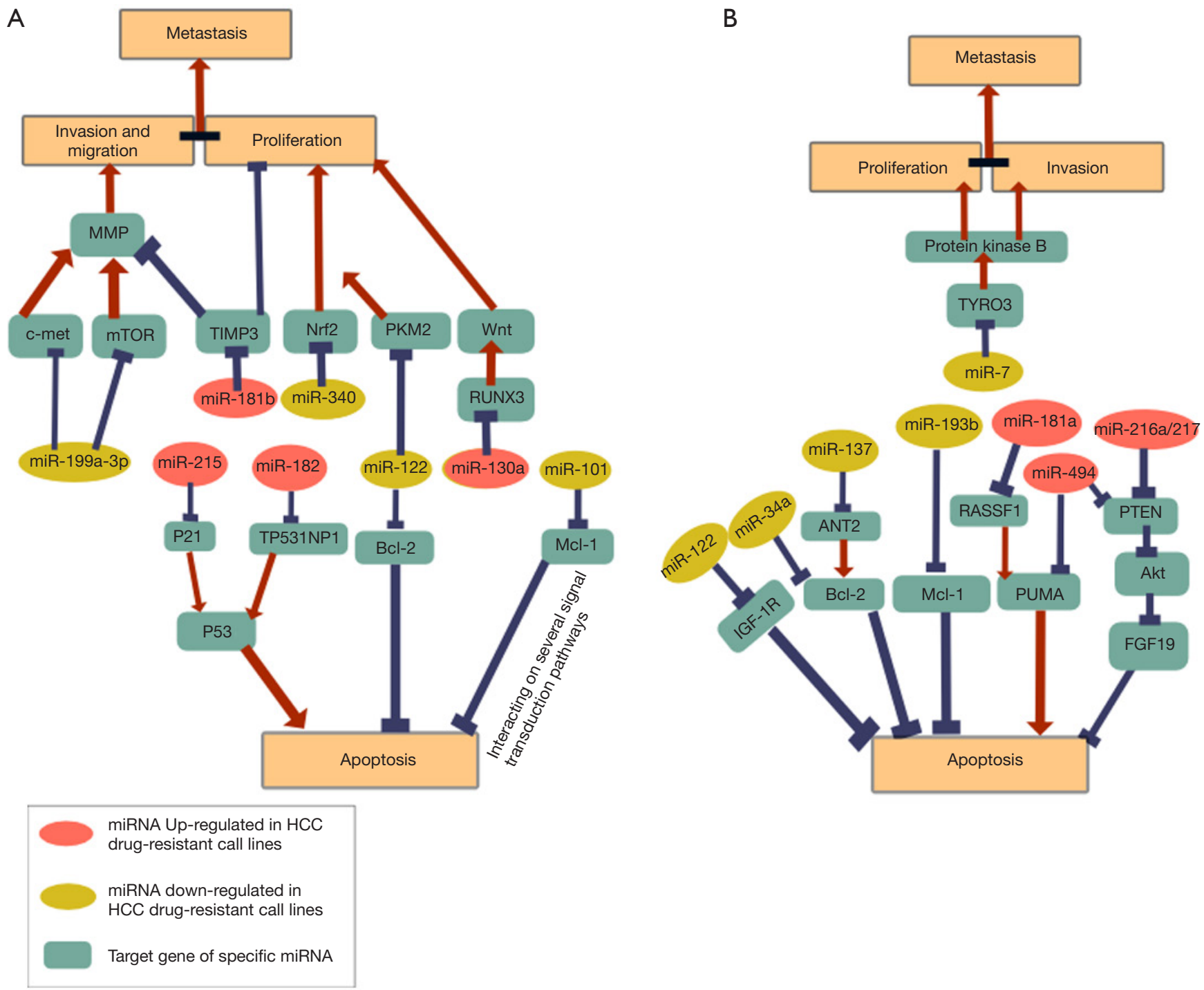

Figure 4 miRNAs involved in multiple pathways contributing to the hallmark of cancer cells in anthracyclines, cisplatin (A) and sorafenib resistance (B).

through the down-regulation of SMAD7, resulting in an EMT phenotype associated with sorafenib resistance (36). This fact points to the involvement of the miR-216a/217SAMD7-TGF- $\beta$ axis in sorafenib resistance in HCC (Figure 3, Figure 5B). Indeed, TGF- $\beta$ has emerged as a potent secreted factor that drives epithelial plasticity leading to EMT, leading to tumor cell dissemination and cancer cell invasion by combining both Smad and non-Smad signaling pathways $(36,84)$.

\section{Apoptosis}

Apoptosis is one of the main pathways involved in the
MDR. In this pathway, one of the key regulators is the tumor suppressor p 53 transcription factor that is activated in response to various cell stress and behavior, including DNA damage. The induction of DNA damage from chemotherapeutic agents may lead to cell cycle arrest, DNA repair, or apoptosis through the $\mathrm{p} 53$ pathway (85). The mutation of $\operatorname{tp} 53$ gene is frequently reported in HCC patient and associated with resistance to chemotherapeutics and poor prognosis (85) due to the alteration of several proapoptotic elements involved in the resistance such as Bax and TRAIL, as well as anti-apoptotic factors including Bcl-2 and Survivin (86-88). Qin et al. reported that the up- 

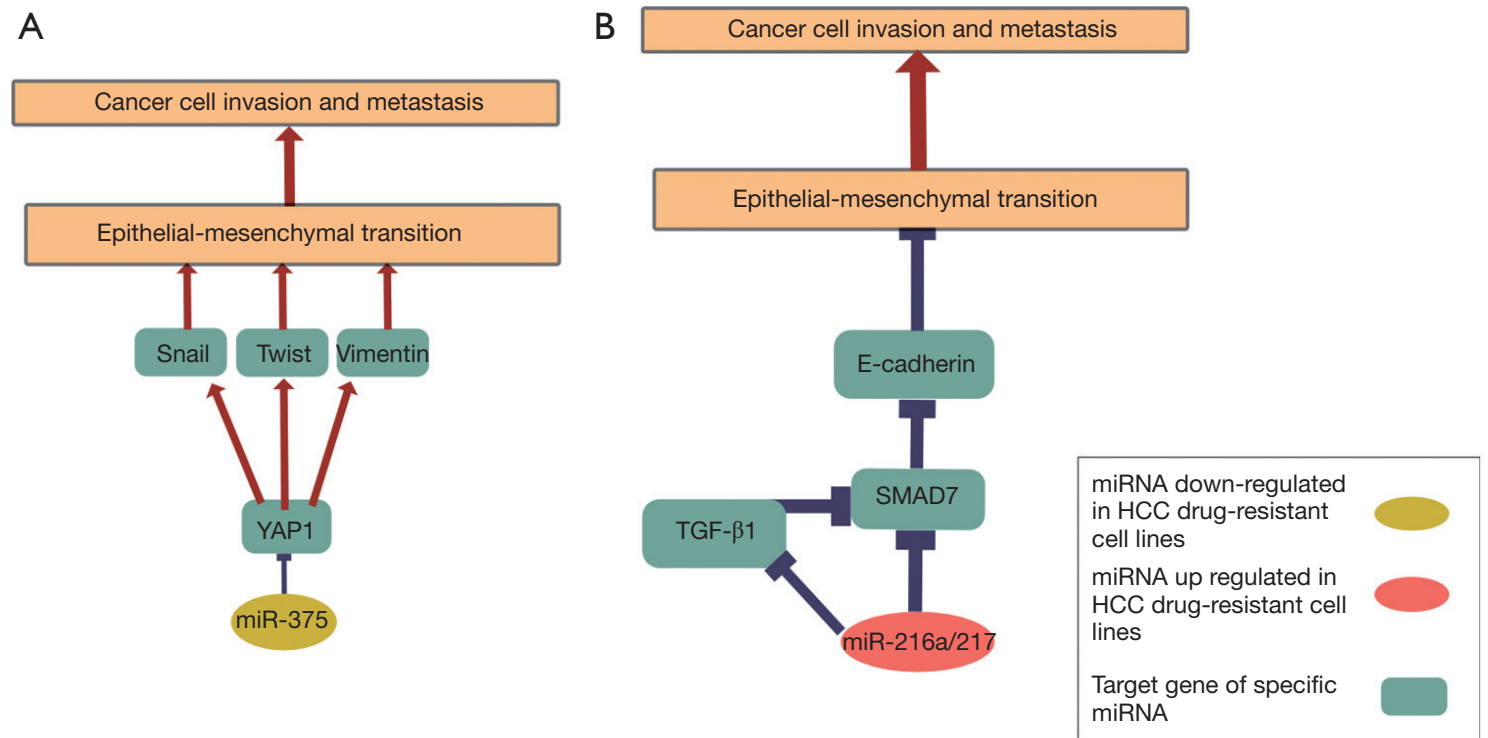

Figure 5 The role of miRNAs in EMT pathway. (A) Down-regulated of miR-375 and miR-145 in anthracyclines and cisplatin-resistant HCC cell lines and their involvement in several pathways related to EMT; (B) up-regulation of mir-216a and miR-217 to SMAD-related pathways of EMT. EMT, epithelial-mesenchymal transition

regulation of miR-182 increased resistance to cisplatin, by inhibiting the expression tumor protein P53 inducible nuclear protein 1 (TP53INP1), a pro-apoptotic gene of the $\mathrm{p} 53$ pathway, leading to the increase of cell viability during treatment with cisplatin (32). This is the only study connecting miRNA, P53 pathway, and drug-resistance, thus more studies on cellular models and clinical specimens are needed to further confirm the hypothesis.

Another miRNA involved in the anti-apoptotic mechanism is miR-101 that have a tumor suppressor role in HCC cell lines. miR-101 acts as a negative regulator of Mcl-1, a critical anti-apoptotic protein in cancer cells that maintains critical element in tumor environments such as growth factor and cellular stress (26). Mcl-1 has been already associated with several drug-resistance phenomenon in various cancers (89) and its downregulation by miR-101 suggests potential application for this miRNA in a Dox resistance setting (26).

During sorafenib treatment, several miRNAs were described to dysregulate the apoptotic pathway contributing to the resistance (Figures 3,4). miR-122 for example, was also significantly reduced in sorafenib-resistant cell lines (42). The mechanism by which this liverspecific miRNA reverses drug resistance involves the downregulation of insulin-like growth factor 1 receptor (IGF-1R) that was proven to inhibits apoptosis and disrupt tolerance to sorafenib in vitro (42).

Two other miRNAs affect apoptosis by regulating the anti-apoptotic proteins Bcl-2 and Mcl-1 in drug resistance. In sorafenib-resistant cell lines, the down-regulation of miR-193b resulted in the overexpression of its target Mcl-1. The transfection with miR-193 mimic suppresses Mcl-1, promoting cell apoptosis, and the co-exposure of miR-193b along with sorafenib had a synergistic effect in treating resistant cell lines (46). The down-regulation of miR-34 and the overexpression of $\mathrm{Bcl}-2$ were concomitantly observed in HCC tissues. Ectopic expression of miR-34a sensitized HCC cells to sorafenib-induced apoptosis through the regulation of $\mathrm{Bcl}-2$ of $\mathrm{Bcl} 2$ and $\mathrm{Mcl}-1$ expression, suggesting an underlying mechanism between miR-34a and anti-apoptotic proteins (44).

The involvement of other miRNAs in the disruption of the apoptotic pathway, a significant event leading to drug resistance, is also known after sorafenib treatment. The down-regulation of miR-137 in sorafenib-resistant cell lines is known to alter adenine nucleotide translocator 2 (ANT2). Its upregulation activates the anti-apoptotic mechanism through the interaction with Bcl2-Bax proteins, ensuring cell survivals $(43,90)$. The restoration of miR-137 was able to reverse the resistance in HCC cell lines showing the potential of this miRNA as anticancer molecule (43). On the other side, the up-regulation of miR-181a in HCC 
cell lines reduced apoptosis by targeting RASSF1, tumor suppressor gene that induces apoptosis through induction of PUMA. Considering that sorafenib inhibits Raf kinase, that is a downstream regulator of RASSF1, the low expression of miR-181 may enhance RASSF1 apoptotic signaling and increase the sensitivity of sorafenib towards cancer cells (40). Considering this evidence, the additional use of these group of miRNAs along with sorafenib might re-sensitize cancer cells to the therapy in drug-resistant settings.

\section{Proliferation, migration, and invasion}

Several miRNAs play an essential role in the mechanisms of tumorigenesis like the invasion and proliferation of drug-resistant cells (Figure 4). Up-regulation of miR-181b in dox-resistant HCC cell lines leads to the inhibition of TIMP metallopeptidase inhibitor 3 (TIMP3), an inhibitor of cells migration, invasion, and angiogenesis in cancer cells (24). In addition, miR-199-3p represses the translation of mTOR and c-Met, suggested as a major activator of MMP, resulting in a reduced cell invasion and metastasis of dox-resistant cells (91). MTOR is also known to play a pivotal role in HCC regulating several other genes involved in major cancer pathways such as STAT3, AKT, HIF- $\alpha$, or ULK1, suggesting its predominant role in multi-drug resistance (92).

Two studies described resistance of cisplatin in HCC cells. $\mathrm{Xu}$ et al. reported that the up-regulation of miR-130a in HuH7-resistant cells directly inhibit the expression of tumor suppressor gene, runt related transcription factor 3 (RUNX3), that results in the activation of $\mathrm{Wnt} / \beta$-catenin, leading to cisplatin resistance (31). In cisplatin-resistant cells, it was reported that miR-340 modulates the expression of nuclear factor, erythroid 2 (Nrf2), a gene involved in drug resistance of various solid tumors and playing a role in cells proliferation and metastasis (34). This study was the first to describe the role of $\mathrm{Nrf2}$, a cytoprotective transcription factor, in the acquisition of chemo-resistance to cancer cells.

Several miRNAs are also regulating the P13K/AKT pathway that is involved in various regulatory mechanism in HCC such as cell proliferation, invasion, apoptosis, metastasis, and autophagy during cancer progression. Kabir et al. identified miR-7 as a potent tumor suppressor targeting tyrosine-protein kinase 3 (TYRO3) that regulates proliferation, migration, and invasion through the $\mathrm{P} 13 /$ AKT pathway in sorafenib-resistant Huh-7 cells model (93). Another study showed that the overexpression of miR-494 in HCC leads to the decrease of PTEN, a multifunctional tumor suppressor that will activate P13/AKT signaling pathway and promoting anti-apoptosis and anti-proliferation in HCC $(39,94)$. miR-494 transfection in HepG2 cells decreases the sensitivity to sorafenib and inversely correlates with the expression of PTEN and apoptosis level. This evidence highlights the importance of miR494/PTEN/P13K/AKT axis in the hallmark of cancer (94). Further, the overexpression of miR-494 decreases also other pro-apoptotic factors such as P27 and PUMA proteins in both cell lines and sorafenib-resistant animal model (39).

\section{Hypoxia}

Hypoxia plays a significant role in enhancing the resistance of cancer cells to cytotoxic drugs (95), particularly relevant in the setting of concurrent induction of acute hypoxia during TACE (50). The hypoxic environment, to which surviving cells are exposed during and soon after chemoembolization, lead to the adaptive mechanisms affecting tumor proliferation, metabolism, and angiogenesis, that involve HIF-1 (96). HIF-1 directly activates various pro-survival genes, such as vascular endothelial growth factor (VEGF), insulin-like growth factor-2 (IGF-2), TGF $\alpha$ and $\beta$, and p53 (96).

Although hypoxia is considered as another leading cause of drug-resistance in cancer, only a single study exists regarding the involvement of miRNAs in this pathway. Indeed, the dysregulation of HIF-1 was associated with sorafenib resistance, being overexpressed in sorafenibresistant HCC specimens (97). In the study, miR-338-3p was observed to be down-regulated in HCC patients and strongly associated with resistance to sorafenib. The reexposure of miR-338-3p to resistant-cell models resensitizes cells by targeting HIF-1 $\alpha$, known to elicit the hypoxic environment in resistant tissue, by enhancing angiogenesis, metabolism, and resistance to apoptosis (45).

\section{Circulating miRNAs as a biomarker to predict resistance to HCC therapy}

The involvement of miRNAs on numerous regulatory networks and mechanisms of drug resistance, and the remarkable specificity of the circulatory expression patterns in cancer settings have raised provocative questions regarding their potential as markers to predict resistance in HCC. Circulating miRNA have been extensively studied as predictive or prognostic biomarkers in several cancer types, including $\mathrm{HCC}(35,41)$. Exosomal miRNAs are believed to represent a sort of cell-to-cell communication in 
which parental cells can transfer molecules, including small RNAs to recipient cells influencing their phenotype (98). In the recent years, evidence pointed out the exosomal transfer of miRNAs to induce chemoresistance in different cancers $(99,100)$. miR-21 was identified as one of the principal players in this setting, with an intriguing role in chemoresistance acquisition in ovarian cancer, neuroblastoma, and lung cancer (100-102). This evidence makes exosomal miRNAs as an essential source of a biomarker for therapy response. Several other small-scale studies have evaluated the role of miRNA as a biomarker for therapy response in several types of cancer. One particular example is found in colon cancer where blood miR-296 predicts tumor progression and metastasis postchemotherapy (103). The serum level of miR-451 in two groups of breast cancer patients that were either sensitive or resistant to neoadjuvant chemotherapy showed a significant difference of expression level, similar with miR-345 taken from the serum of advanced rectal cancer patient treated with preoperative chemoradiotherapy (CRT) $(104,105)$. These studies open the question whether miRNAs might also show a similar potential in predicting therapeutic response in HCC.

However, only a few studies reported evidence on the role of circulating miRNAs as therapy response biomarker in HCC. Liu et al. evaluated serum samples from 136 HCC patient undergoing TACE (a mixture of dox, lipiodol, and contrast agent) describing the potential role of miR$200 \mathrm{a}$ as an independent prognostic factor associated with disease outcome (106). Serum miR-335 is also significantly correlated with the therapeutic response after TACE in 125 HCC patients (107) in which the low miR-335 levels were significantly associated with inadequate treatment response and decreased survival. Conversely, higher expression of miR-590-5p had been related to a better response after TACE in patients with HCC (58). However, the latter study is a retrospective study with a small sample size that needs further validation to assess its potential and its regulatory role in the mechanism of multi-drug resistance in HCC.

Among the several limitations of the study in this area, one particular miRNA was assessed in regard to the expression in HCC patients, its association with therapy response, and its mechanism for regulating the drugresistance. Kim et al. described the association of high expression of plasma miR-122 with early TACE (dox) refractoriness in 177 HCC patients (108). This is in line with the role of miR-122 in multi-drug resistance as this miRNA is strongly associated with multiple pathways regulating apoptosis, drug accumulation, invasion, and proliferation in drug-resistant HCC cells.

Meanwhile, in, there is only one small-scale study addressing the potential of miRNA as a predictor of response to sorafenib. High expression of serum miR-181a-5p in HCC patients treated with the drug was associated with better disease control and response to treatment (109). These findings, although limited by the small-sized cohort, are in line with the participation or miR-181 in cellular mechanisms leading to sorafenib resistance through EMT (110).

\section{Conclusions}

Numerous studies addressed the highly complex mechanism(s) of multi-drug resistance in HCC, which remains the main obstacle to achieving the success of chemotherapies. The development of MDR involves multiple pathways and networks, with diverse mechanisms and subtle key players. In the present review, we summarized the regulatory role of miRNAs in modulating drug resistance to anthracycline, cisplatin, and the multikinase inhibitor sorafenib. miRNAs play a significant role in targeting specific genes involved in the resistance, as many of them target multiple genes involved in different but interpolated pathways. Various studies prove that counterbalance the expression of miRNAs in resistant cells can re-sensitize cancer cells to chemotherapeutic agents, strengthening the potential of miRNAs as co-adjuvants in anticancer therapy.

However, there are still several technical obstacles to translate these results into a convincing clinical application. A homogenous methodological protocol to process and analyze sample has not yet been reached, making the comparison of different studies at least tenuous. In addition, the small cohorts and the heterogeneity of the disease limit the transposition of the results from bench to bedside. Indeed, the potential of miRNA is undeniable, and their paramount role in drug resistance could be utilized as alternatives in the future as well as a promising circulatory biomarker predicting therapy response. However, several additional evidence must be provided before miRNA will enter the routine clinical scenario.

\section{Acknowledgments}

Funding: MY Pratama is supported by fellowships from the Lembaga Pengelola Dana Pendidikan (LPDP) of the Indonesian Ministry of Finance. The study was partially 
supported by an intramural grant from the Italian Liver Foundation and by grant POR-FESR 2014-2020 axis 1 activity 1.3 approved with DGR n4526 16/6/17, "Surface Enhanced Raman microRNA per la diagnosi e Management del Cancro (SERMI4CANCER)".

\section{Footnote}

Conflicts of Interest: The authors have no conflicts of interest to declare.

Ethical Statement: The authors are accountable for all aspects of the work in ensuring that questions related to the accuracy or integrity of any part of the work are appropriately investigated and resolved.

\section{References}

1. World Health Organization. GLOBOCAN 2012 cancer fact sheets: liver cancer. World Health Organization; 2012. Available online: http://globocan.iarc.fr/Pages/fact_sheets_ cancer.aspx? cancer $=$ liver

2. European Association For The Study Of The Liver, European Organisation For Research And Treatment Of Cancer. EASL-EORTC clinical practice guidelines: management of hepatocellular carcinoma. J Hepatol 2012;56:908-43.

3. Imai $N$, Ishigami $M$, Ishizu $Y$, et al. Transarterial chemoembolization for hepatocellular carcinoma: A review of techniques. World J Hepatol 2014;6:844-50.

4. Lanza E, Donadon M, Poretti D, et al. Transarterial Therapies for Hepatocellular Carcinoma. Liver Cancer 2016;6:27-33.

5. Malagari K, Pomoni M, Kelekis A, et al. Prospective Randomized Comparison of Chemoembolization with Doxorubicin-Eluting Beads and Bland Embolization with BeadBlock for Hepatocellular Carcinoma. Cardiovasc Intervent Radiol 2010;33:541-51.

6. Sanoff HK, Chang Y, Stavas JM, et al. Effectiveness of Initial Transarterial Chemoembolization for Hepatocellular Carcinoma Among Medicare Beneficiaries. J Natl Compr Canc Netw 2015;13:1102-10.

7. Liu X, Wang Z, Chen Z, et al. Efficacy and Safety of Transcatheter Arterial Chemoembolization and Transcatheter Arterial Chemotherapy Infusion in Hepatocellular Carcinoma: A Systematic Review and Meta-Analysis. Oncol Res 2018 Mar 5;26:231-9.

8. Boily G, Villeneuve J-P, Lacoursière L, et al. Transarterial embolization therapies for the treatment of hepatocellular carcinoma: CEPO review and clinical recommendations. HPB 2015;17:52-65.

9. Llovet JM, Ricci S, Mazzaferro V, et al. Sorafenib in advanced hepatocellular carcinoma. $\mathrm{N}$ Engl J Med 2008;359:378-90.

10. Miyahara K, Nouso K, Yamamoto K. Chemotherapy for advanced hepatocellular carcinoma in the sorafenib age. World J Gastroenterol 2014;20:4151-9.

11. Wilhelm SM, Adnane L, Newell P, et al. Preclinical overview of sorafenib, a multikinase inhibitor that targets both Raf and VEGF and PDGF receptor tyrosine kinase signaling. Mol Cancer Ther 2008;7:3129-40.

12. Liu L, Cao Y, Chen C, et al. Sorafenib blocks the RAF/ MEK/ERK pathway, inhibits tumor angiogenesis, and induces tumor cell apoptosis in hepatocellular carcinoma model PLC/PRF/5. Cancer Res 2006;66:11851-8.

13. Chen Y, Liu YC, Sung YC, et al. Overcoming sorafenib evasion in hepatocellular carcinoma using CXCR4targeted nanoparticles to co-deliver MEK-inhibitors. Sci Rep 2017;7:44123.

14. Zhu YJ, Zheng B, Wang HY, et al. New knowledge of the mechanisms of sorafenib resistance in liver cancer. Acta Pharmacol Sin 2017;38:614-22.

15. Le Grazie M, Biagini MR, Tarocchi M, et al. Chemotherapy for hepatocellular carcinoma: The present and the future. World J Hepatol 2017;9:907-20.

16. Lee RC, Feinbaum RL, Ambros V. The C. elegans heterochronic gene lin-4 encodes small RNAs with antisense complementarity to lin-14. Cell 1993;75:843-54.

17. Lopez-Serra P, Esteller M. DNA methylation-associated silencing of tumor-suppressor microRNAs in cancer. Oncogene 2012;31:1609-22.

18. Loginov VI, Rykov SV, Fridman MV, et al. Methylation of miRNA genes and oncogenesis. Biochemistry (Mosc) 2015;80:145-62.

19. Calin GA, Dumitru CD, Shimizu M, et al. Frequent deletions and down-regulation of micro- RNA genes miR15 and miR16 at 13q14 in chronic lymphocytic leukemia. Proc Natl Acad Sci U S A 2002;99:15524-9.

20. Johnson SM, Grosshans H, Shingara J, et al. RAS is regulated by the let-7 microRNA family. Cell 2005;120:635-47.

21. Bajan S, Hutvagner G. Regulation of miRNA processing and miRNA mediated gene repression in cancer. Microrna 2014;3:10-7.

22. He L, Thomson JM, Hemann MT, et al. A microRNA polycistron as a potential human oncogene. Nature 
2005;435:828-33.

23. Murakami Y, Yasuda T, Saigo K, et al. Comprehensive analysis of microRNA expression patterns in hepatocellular carcinoma and non-tumorous tissues. Oncogene 2006;25:2537-45.

24. Wang B, Hsu SH, Majumder S, et al. TGFbetamediated upregulation of hepatic miR-181b promotes hepatocarcinogenesis by targeting TIMP3. Oncogene 2010;29:1787-97.

25. Yahya SMM, Fathy SA, El-Khayat ZA, et al. Possible Role of microRNA-122 in Modulating Multidrug Resistance of Hepatocellular Carcinoma. Indian J Clin Biochem 2018;33:21-30.

26. He H, Tian W, Chen H, et al. MicroRNA-101 sensitizes hepatocellular carcinoma cells to doxorubicininduced apoptosis via targeting Mcl-1. Mol Med Rep 2016;13:1923-9.

27. Jin F, Wang Y, Li M, et al. miR-26 enhances chemosensitivity and promotes apoptosis of hepatocellular carcinoma cells through inhibiting autophagy. Cell Death Dis 2017;8:e2540.

28. Xue H, Yu Z, Liu Y, et al. Delivery of miR-375 and doxorubicin hydrochloride by lipid-coated hollow mesoporous silica nanoparticles to overcome multiple drug resistance in hepatocellular carcinoma. Int J Nanomedicine 2017;12:5271-87.

29. Yang T, Zheng ZM, Li XN, et al. miR-223 modulates multidrug resistance via downregulation of ABCB1 in hepatocellular carcinoma cells. Exp Biol Med (Maywood) 2013;238:1024-32.

30. Gao AM, Zhang XY, Hu JN, et al. Apigenin sensitizes hepatocellular carcinoma cells to doxorubic through regulating miR-520b/ATG7 axis. Chem Biol Interact 2018;280:45-50.

31. Xu N, Shen C, Luo Y, et al. Upregulated miR-130a increases drug resistance by regulating RUNX3 and Wnt signaling in cisplatin-treated HCC cell. Biochem Biophys Res Commun 2012;425:468-72.

32. Qin J, Luo M, Qian H, et al. Upregulated miR-182 increases drug resistance in cisplatin-treated HCC cell by regulating TP53INP1. Gene 2014;538:342-7.

33. Pan C, Wang X, Shi K, et al. miR-122 Reverses the Doxorubicin-Resistance in Hepatocellular Carcinoma Cells through Regulating the Tumor Metabolism. PLoS One 2016;11:e0152090.

34. Shi L, Chen ZG, Wu LL, et al. miR-340 reverses cisplatin resistance of hepatocellular carcinoma cell lines by targeting Nrf2-dependent antioxidant pathway. Asian Pac
J Cancer Prev 2014;15:10439-44.

35. Tsuchiya N, Sawada Y, Endo I, et al. Biomarkers for the early diagnosis of hepatocellular carcinoma. World J Gastroenterol 2015;21:10573-83.

36. Xia H, Ooi LLPJ, Hui KM. MicroRNA-216a/217induced epithelial-mesenchymal transition targets PTEN and SMAD7 to promote drug resistance and recurrence of liver cancer. Hepatology 2013;58:629-41.

37. Liu K, Liu S, Zhang W, et al. miR-222 regulates sorafenib resistance and enhance tumorigenicity in hepatocellular carcinoma. Int J Oncol 2014;45:1537-46.

38. Tang S, Tan G, Jiang X, et al. An artificial lncRNA targeting multiple miRNAs overcomes sorafenib resistance in hepatocellular carcinoma cells. Oncotarget 2016;7:73257-69.

39. Pollutri D, Patrizi C, Marinelli S, et al. The epigenetically regulated miR-494 associates with stem-cell phenotype and induces sorafenib resistance in hepatocellular carcinoma. Cell Death Dis 2018;9:4.

40. Azumi J, Tsubota T, Sakabe T, et al. miR-181a induces sorafenib resistance of hepatocellular carcinoma cells through downregulation of RASSF1 expression. Cancer Sci 2016;107:1256-62.

41. Zhang K, Chen J, Zhou H, et al. PU.1/microRNA-142$3 \mathrm{p}$ targets ATG5/ATG16L1 to inactivate autophagy and sensitize hepatocellular carcinoma cells to sorafenib. Cell Death Dis 2018;9:312.

42. Xu Y, Huang J, Ma L, et al. MicroRNA-122 confers sorafenib resistance to hepatocellular carcinoma cells by targeting IGF-1R to regulate RAS/RAF/ERK signaling pathways. Cancer Lett 2016;371:171-81.

43. Lu AQ, Lv B, Qiu F, et al. Upregulation of miR-137 reverses sorafenib resistance and cancer-initiating cell phenotypes by degrading ANT2 in hepatocellular carcinoma. Oncol Rep 2017;37:2071-8.

44. Yang F, Li Q, Gong Z, et al. MicroRNA-34a targets Bcl-2 and sensitizes human hepatocellular carcinoma cells to sorafenib treatment. Technol Cancer Res Treat 2014;13:77-86.

45. Xu H, Zhao L, Fang Q, et al. miR-338-3p inhibits hepatocarcinoma cells and sensitizes these cells to sorafenib by targeting hypoxia-induced factor $1 \alpha$. PLoS One 2014;9:e115565.

46. Mao K, Zhang J, He C, et al. Restoration of miR-193b sensitizes Hepatitis B virus-associated hepatocellular carcinoma to sorafenib. Cancer Lett 2014;352:245-52.

47. Cox J, Weinman S. Mechanisms of doxorubicin resistance in hepatocellular carcinoma. Hepat Oncol 2016;3:57-9. 
48. Vasiliou V, Vasiliou K, Nebert DW. Human ATP-binding cassette (ABC) transporter family. Hum Genomics 2009;3:281-90.

49. $\mathrm{Ng}$ IO, Liu CL, Fan ST, Ng M. Expression of P-glycoprotein in hepatocellular carcinoma. A determinant of chemotherapy response. Am J Clin Pathol 2000;113:355-63.

50. Asghar U, Meyer T. Are there opportunities for chemotherapy in the treatment of hepatocellular cancer? J Hepatol 2012;56:686-95.

51. Borel F, Han R, Visser A, et al. Adenosine triphosphatebinding cassette transporter genes up-regulation in untreated hepatocellular carcinoma is mediated by cellular microRNAs. Hepatology 2012;5 5:821-32.

52. Wen DY, Huang JC, Wang JY, et al. Potential clinical value and putative biological function of miR-122-5p in hepatocellular carcinoma: A comprehensive study using microarray and RNA sequencing data. Oncol Lett 2018;16:6918-29.

53. Bandiera S, Pfeffer S, Baumert TF, et al. miR-122--a key factor and therapeutic target in liver disease. J Hepatol 2015;62:448-57.

54. Tsai WC, Hsu SD, Hsu CS, et al. MicroRNA-122 plays a critical role in liver homeostasis and hepatocarcinogenesis. J Clin Invest 2012;122:2884-97.

55. Xu Y, Xia F, Ma L, et al. MicroRNA-122 sensitizes HCC cancer cells to adriamycin and vincristine through modulating expression of MDR and inducing cell cycle arrest. Cancer Lett 2011;310:160-9.

56. Fan YP, Liao JZ, Lu YQ, et al. miR-375 and Doxorubicin Co-delivered by Liposomes for Combination Therapy of Hepatocellular Carcinoma. Mol Ther Nucleic Acids 2017;7:181-9.

57. Zhao P, Wu S, Cheng Y, et al. miR-375 delivered by lipidcoated doxorubicin-calcium carbonate nanoparticles overcomes chemoresistance in hepatocellular carcinoma. Nanomedicine 2017;13:2507-16.

58. Chen M, Wu L, Tu J, et al. miR-590-5p suppresses hepatocellular carcinoma chemoresistance by targeting YAP1 expression. EBioMedicine 2018;35:142-54.

59. You L, Jin S, Zhu L, et al. Autophagy, autophagyassociated adaptive immune responses and its role in hematologic malignancies. Oncotarget 2017;8:12374-88.

60. Amaravadi R, Kimmelman AC, White E. Recent insights into the function of autophagy in cancer. Genes Dev 2016;30:1913-30.

61. White E, DiPaola RS. The double-edged sword of autophagy modulation in cancer. Clin Cancer Res
2009;15:5308-16.

62. Sui X, Chen R, Wang Z, et al. Autophagy and chemotherapy resistance: a promising therapeutic target for cancer treatment. Cell Death Dis 2013;4:e838.

63. Hu YL, Jahangiri A, DeLay M, et al. Tumor cell autophagy as an adaptive response mediating resistance to treatments like anti-angiogenic therapy. Cancer Res 2012;72:4294-9.

64. Lazarus MB, Novotny CJ, Shokat KM. Structure of the human autophagy initiating kinase ULK1 in complex with potent inhibitors. ACS Chem Biol 2015;10:257-61.

65. Funderburk SF, Wang QJ, Yue Z. The Beclin 1-VPS34 complex--at the crossroads of autophagy and beyond. Trends Cell Biol 2010;20:355-62.

66. Chang Y, Yan W, He X, et al. miR-375 inhibits autophagy and reduces viability of hepatocellular carcinoma cells under hypoxic conditions. Gastroenterology 2012;143:17787.e8.

67. Shimizu S, Takehara T, Hikita H, et al. Inhibition of autophagy potentiates the antitumor effect of the multikinase inhibitor sorafenib in hepatocellular carcinoma. Int J Cancer 2012;131:548-57.

68. Shi YH, Ding ZB, Zhou J, et al. Targeting autophagy enhances sorafenib lethality for hepatocellular carcinoma via ER stress-related apoptosis. Autophagy 2011;7:1159-72.

69. Zhai B, Hu F, Jiang X, et al. Inhibition of Akt reverses the acquired resistance to sorafenib by switching protective autophagy to autophagic cell death in hepatocellular carcinoma. Mol Cancer Ther 2014;13:1589-98.

70. Honma Y, Shimizu S, Takehara T, et al. Sorafenib enhances proteasome inhibitor-induced cell death via inactivation of Akt and stress-activated protein kinases. J Gastroenterol 2014;49:517-26.

71. Manov I, Pollak Y, Broneshter R, et al. Inhibition of doxorubicin-induced autophagy in hepatocellular carcinoma Hep3B cells by sorafenib--the role of extracellular signal-regulated kinase counteraction. FEBS J 2011;278:3494-507.

72. Mao B, Wang G. MicroRNAs involved with hepatocellular carcinoma (Review). Oncol Rep 2015;34:2811-20.

73. He C, Dong X, Zhai B, et al. miR-21 mediates sorafenib resistance of hepatocellular carcinoma cells by inhibiting autophagy via the PTEN/Akt pathway. Oncotarget 2015;6:28867-81.

74. Sini P, James D, Chresta C, et al. Simultaneous inhibition of mTORC1 and mTORC2 by mTOR kinase inhibitor AZD8055 induces autophagy and cell death in cancer cells. Autophagy 2010;6:553-4.

75. Yoon EL, Yeon JE, Ko E, et al. An Explorative Analysis for 
the Role of Serum miR-10b-3p Levels in Predicting Response to Sorafenib in Patients with Advanced Hepatocellular Carcinoma. J Korean Med Sci 2017;32:212-20.

76. Lamouille S, Xu J, Derynck R. Molecular mechanisms of epithelial-mesenchymal transition. Nat Rev Mol Cell Biol 2014;15:178-96.

77. Thiery JP, Acloque H, Huang RYJ, et al. Epithelialmesenchymal transitions in development and disease. Cell 2009;139:871-90.

78. Du B, Shim JS. Targeting Epithelial-Mesenchymal Transition (EMT) to Overcome Drug Resistance in Cancer. Molecules 2016;21. doi: 10.3390/ molecules21070965.

79. Thiery JP. Epithelial-mesenchymal transitions in tumour progression. Nat Rev Cancer 2002;2:442-54.

80. Translating epithelial mesenchymal transition markers into the clinic: Novel insights from proteomics - ScienceDirect. Available online: https://www.sciencedirect.com/science/ article/pii/S2212968516300034

81. Liu Y, He K, Hu Y, et al. YAP modulates TGF- $\beta 1$-induced simultaneous apoptosis and EMT through upregulation of the EGF receptor. Sci Rep 2017;7:45523.

82. van Malenstein H, Dekervel J, Verslype C, et al. Longterm exposure to sorafenib of liver cancer cells induces resistance with epithelial-to-mesenchymal transition, increased invasion and risk of rebound growth. Cancer Lett 2013;329:74-83.

83. Bae JS, Noh SJ, Kim KM, et al. Serum response factor induces epithelial to mesenchymal transition with resistance to sorafenib in hepatocellular carcinoma. Int J Oncol 2014;44:129-36.

84. Katsuno Y, Lamouille S, Derynck R. TGF- $\beta$ signaling and epithelial-mesenchymal transition in cancer progression. Curr Opin Oncol 2013;25:76.

85. Rivlin N, Brosh R, Oren M, et al. Mutations in the p53 Tumor Suppressor Gene: Important Milestones at the Various Steps of Tumorigenesis. Genes Cancer 2011;2:466-74.

86. Hoffman WH, Biade S, Zilfou JT, et al. Transcriptional repression of the anti-apoptotic survivin gene by wild type p53. J Biol Chem 2002;277:3247-57.

87. Wu Y, Mehew JW, Heckman CA, et al. Negative regulation of bcl-2 expression by p53 in hematopoietic cells. Oncogene 2001;20:240-51.

88. Hu Y, Xu K, Yagüe E. miR-218 targets survivin and regulates resistance to chemotherapeutics in breast cancer. Breast Cancer Res Treat 2015;151:269-80.

89. Quinn BA, Dash R, Azab B, et al. Targeting Mcl-1 for the therapy of cancer. Expert Opin Investig Drugs 2011;20:1397-411.

90. Chevrollier A, Loiseau D, Reynier P, et al. Adenine nucleotide translocase 2 is a key mitochondrial protein in cancer metabolism. Biochim Biophys Acta 2011;1807:562-7.

91. Fornari F, Milazzo M, Chieco P, et al. miR-199a-3p regulates $\mathrm{mTOR}$ and $\mathrm{c}-\mathrm{Met}$ to influence the doxorubicin sensitivity of human hepatocarcinoma cells. Cancer Res 2010;70:5184-93.

92. Matter MS, Decaens T, Andersen JB, et al. Targeting the mTOR pathway in hepatocellular carcinoma: Current state and future trends. J Hepatol 2014;60:855-65.

93. Kabir TD, Ganda C, Brown RM, et al. A microRNA-7/ growth arrest specific 6/TYRO3 axis regulates the growth and invasiveness of sorafenib-resistant cells in human hepatocellular carcinoma. Hepatology 2018;67:216-31.

94. Liu K, Liu S, Zhang W, et al. miR-494 promotes cell proliferation, migration and invasion, and increased sorafenib resistance in hepatocellular carcinoma by targeting PTEN. Oncol Rep 2015;34:1003-10.

95. Sciarra A, Ronot M, Di Tommaso L, et al. TRIP: a pathological score for transarterial chemoembolization resistance individualized prediction in hepatocellular carcinoma. Liver Int 2015;35:2466-73.

96. Luo D, Wang Z, Wu J, et al. The Role of Hypoxia Inducible Factor-1 in Hepatocellular Carcinoma. BioMed Res Int 2014. Available online: https://www.ncbi.nlm.nih. gov/pmc/articles/PMC4101982/

97. Liang Y, Zheng T, Song R, et al. Hypoxia-mediated sorafenib resistance can be overcome by EF24 through Von Hippel-Lindau tumor suppressor-dependent HIF$1 \alpha$ inhibition in hepatocellular carcinoma. Hepatology 2013;57:1847-57.

98. Kogure T, Lin WL, Yan IK, et al. Intercellular nanovesicle-mediated microRNA transfer: a mechanism of environmental modulation of hepatocellular cancer cell growth. Hepatology 2011;54:1237-48.

99. Vietsch EE, van Eijck CH, Wellstein A. Circulating DNA and Micro-RNA in Patients with Pancreatic Cancer. Pancreat Disord Ther 2015;5(2).

100.Xiao X, Yu S, Li S, et al. Exosomes: decreased sensitivity of lung cancer A549 cells to cisplatin. PLoS One 2014;9:e89534.

101. Challagundla KB, Wise PM, Neviani P, et al. Exosomemediated transfer of microRNAs within the tumor microenvironment and neuroblastoma resistance to chemotherapy. J Natl Cancer Inst 2015. doi: 10.1093/jnci/ djv135. 
102.Au Yeung CL, Co NN, Tsuruga T, et al. Exosomal transfer of stroma-derived miR2 1 confers paclitaxel resistance in ovarian cancer cells through targeting APAF1. Nat Commun 2016;7:11150.

103. Shivapurkar N, Mikhail S, Navarro R, et al. Decrease in blood miR-296 predicts chemotherapy resistance and poor clinical outcome in patients receiving systemic chemotherapy for metastatic colon cancer. Int J Colorectal Dis 2013;28:887.

104.Gu X, Xue JQ, Han SJ, et al. Circulating microRNA-451 as a predictor of resistance to neoadjuvant chemotherapy in breast cancer. Cancer Biomark 2016;16:395-403.

105.Yu J, Li N, Wang X, et al. Circulating serum microRNA-345 correlates with unfavorable pathological response to preoperative chemoradiotherapy in locally advanced rectal cancer. Oncotarget 2016;7:64233-43.

106. Liu M, Liu J, Wang L, et al. Association of serum microRNA expression in hepatocellular carcinomas treated with transarterial chemoembolization and patient survival.
PLoS One 2014;9:e109347.

107. Cui L, Hu Y, Bai B, et al. Serum miR-335 Level is Associated with the Treatment Response to TransArterial Chemoembolization and Prognosis in Patients with Hepatocellular Carcinoma. Cell Physiol Biochem 2015;37:276-83.

108. Kim SS, Nam JS, Cho HJ, et al. Plasma micoRNA-122 as a predictive marker for treatment response following transarterial chemoembolization in patients with hepatocellular carcinoma. J Gastroenterol Hepatol 2017;32:199-207.

109. Nishida N, Arizumi T, Hagiwara S, et al. MicroRNAs for the Prediction of Early Response to Sorafenib Treatment in Human Hepatocellular Carcinoma. Liver Cancer 2017;6:113-25.

110.Meng F, Glaser SS, Francis H, et al. Functional analysis of microRNAs in human hepatocellular cancer stem cells. J Cell Mol Med 2012;16:160-73.

Cite this article as: Pratama MY, Pascut D, Massi MN, Tiribelli C. The role of microRNA in the resistance to treatment of hepatocellular carcinoma. Ann Transl Med 2019;7(20):577. doi: 10.21037/atm.2019.09.142 